This is a pre-copy-editing, author-produced PDF of an article accepted for publication in Osteoporosis International following peer review. The original publication is available at www.springerlink.com

\title{
Cardiovascular Effects of Calcium Supplementation
}

\author{
Ian R Reid ${ }^{1}$ \\ Mark J Bolland ${ }^{1}$ \\ Alison Avenell ${ }^{2}$ \\ Andrew Grey ${ }^{1}$ \\ 1. Department of Medicine \\ Faculty of Medical and Health Sciences \\ University of Auckland \\ Auckland, New Zealand \\ 2. Health Services Research Unit \\ University of Aberdeen \\ Foresterhill, Aberdeen AB25 2ZD, Scotland
}

Correspondence to:

Professor Ian Reid

Faculty of Medical and Health Sciences

University of Auckland

Private Bag 92019

Auckland

New Zealand

Tel: (+64 9) 3737599 extn 86259

Fax: (+64 9) 3082308

email: i.reid@auckland.ac.nz

Supported by the Health Research Council of New Zealand. AA was funded by a Career Scientist award of the Chief Scientist Office of the Scottish Government Health Directorates. The Health Services Research Unit is funded by the Chief Scientist Office of the Scottish Government Health Directorates.

Key words: osteoporosis, mineral supplements, nutrition 


\section{Abstract}

Trials in normal older women and in patients with renal impairment suggest that calcium supplements increase the risk of cardiovascular disease. To further assess their safety, we recently conducted a meta-analysis of trials of calcium supplements, and found a $27-31 \%$ increase in risk of myocardial infarction, and a $12-20 \%$ increase in risk of stroke. These findings are robust because they are based on pre-specified analyses of randomized, placebo-controlled trials and show consistent risk across the trials. The fact that cardiovascular events were not primary endpoints of any of these studies will introduce noise but not bias into the data. A recent re-analysis of the Women's Health Initiative suggests that co-administration of vitamin D with calcium does not lessen these adverse effects.

The increased cardiovascular risk with calcium supplements is consistent with epidemiological data relating higher circulating calcium concentrations to cardiovascular disease in normal populations. There are several possible pathophysiological mechanisms for these effects, including effects on vascular calcification, on the function of vascular cells, and on blood coagulation. Calciumsensing receptors might mediate some of these effects.

Because calcium supplements produce small reductions in fracture risk and a small increase in cardiovascular risk, there may be no net benefit from their use. Food sources of calcium appear to produce similar benefits on bone density although their effects on fracture are unclear. Since food sources have not been associated with adverse cardiovascular effects, they may be preferable. Available evidence suggests that other osteoporosis treatments are still effective without calcium coadministration. 


\section{Introduction}

The concept that the use of calcium supplements might impact on the incidence of heart disease dates back at least as far as the 1960s, when beneficial effects of calcium loading on circulating cholesterol levels were suggested [1]. The proposed mechanisms for this effect are binding of lipids and bile acids by calcium in the gut interfering with fat absorption [2-4], and possible indirect effects of vitamin D metabolites on lipid metabolism [5]. The calcium-cholesterol connection has been examined more rigorously since that time, with mixed results from randomized trials $[3,6-8]$. There has been more extensive investigation of the effects of calcium supplements on blood pressure with a fairly consistent demonstration of average decrements of $1-2 \mathrm{mmHg}$ in both systolic and diastolic pressures [9-11] in those allocated to calcium, and these effects may be greater in subjects with low dietary calcium intakes [8]. These findings, together with the possible inverse association between calcium intake and body weight [12], led to the suggestion that calcium supplements might decrease the incidence of cardiovascular events. Running counter to this, however, has been evidence from patients with renal failure, in whom cardiovascular disease is one of the principal drivers of morbidity and mortality. While the presence of hyperphosphatemia in renal failure renders this a somewhat different situation from the normal, older postmenopausal women, there is accumulating evidence that the use of calcium salts as phosphate binders in both intermediate [13] and advanced [14] renal failure is associated with accelerated arterial calcification and increased mortality,. This has led to reduced use of noncalcium phosphate binders in nephrology practice.

\section{Auckland Calcium Study}

In the light of the above evidence, we pre-specified several cardiovascular events as secondary endpoints in a prospective, randomized, placebo-controlled trial of calcium supplementation ( $1 \mathrm{~g}$ per day as the citrate) in 1471 normal, late postmenopausal women (mean age 74 years). Contrary to our hypothesis of benefit, the subjects' self-reports of adverse events showed a two-fold excess of myocardial infarctions (MI) and a 44\% increase in the risk of stroke (non-significant) [15]. Because of this unexpected outcome and the potential importance of this issue, we collaborated with a cardiologist and a neurologist to formally adjudicate all of these events, and used a national register of hospital admissions to maximize event capture. The adjudication process resulted in some changes in the numbers of individual events but the increased risk in the calcium group remained, there being a $49 \%$ excess in numbers of women with myocardial infarction $(P=0.16)$ and a $43 \%$ increase in the rate ratio of the pre-specified composite endpoint of myocardial infarction, stroke or sudden death $(P=0.04)[15]$.

These findings raised a major concern regarding the safety of calcium supplements but did not provide definitive evidence. The conventional response to potentially important findings of borderline statistical significance is to conduct a definitive trial. Such a study, powered to detect a $20 \%$ difference in cardiovascular events between calcium and placebo groups, would need $>20,000$ participants followed for $>5$ years. While this is theoretically feasible, the ethical and recruitment problems that arise from testing a hypothesis of harm make this effectively impossible. Therefore, the only practical way to further address this question was to access data on cardiac safety from existing trials, in the hope that an adequately powered meta-analysis might be possible. 


\section{Meta-analysis of Calcium Supplements and Vascular Events}

We have recently published such an analysis of adverse event data from randomized controlled trials of calcium supplements [16]. Having drafted a protocol for this metaanalysis, we contacted the principal investigators of the 15 eligible trials inviting their participation and comments on the protocol. Suggested amendments were incorporated into the protocol, which was reviewed and approved by all investigators participating in the patient-level meta-analysis before data collation commenced. The intent was to collect patient-level data on cardiovascular events that occurred during each of the studies. Where such data were not available, trial-level summary data were requested. Of 15 eligible trials, cardiovascular data were not available in 4, patient-level data were available from 5 (8,151 participants, median follow-up 3.6y) and trial-level data were available from 11 (11,921 participants, median follow-up 4y). In 10 of the 11 trials providing data for analysis, calcium supplements were studied at a dose of at least $1000 \mathrm{mg}$ daily. The average age of participants was 73y, and 83\% were women. Thus, the participants were similar demographically to the group within the general population which is most commonly prescribed calcium supplements. Cardiovascular disease was not a primary endpoint of any of the trials, and events were ascertained variously by participants' self-reports, hospital admission data, and death certificates. The pre-specified primary endpoints of the analysis were time to first MI, time to first stroke, and time to first event for the composite endpoint of MI, stroke, or sudden death.

The results of the meta-analysis of both individual patient-level and trial-level data are summarized in Figure 1. In both analyses, allocation to calcium supplements conferred a significant, approximately $30 \%$, increase in the risk of $\mathrm{MI}$, and smaller, non-significant increases in the risks of stroke, the composite of MI, stroke and sudden death, and total mortality. The individual-patient level analysis provides an insight into the timing of the adverse effect of calcium supplements, and suggests that the increase in risk of $\mathrm{Ml}$ begins soon after commencing treatment. The results of the trial-level analysis, incorporating data from 11 trials, demonstrate a striking consistency of effect in those studies that reported a substantial number of events. Calcium supplementation also tended to increase the risk of recurrent events, so that the relative risks of total events were increased by $24-32 \%$.

\section{Responses to Meta-Analysis}

As expected, the meta-analysis has caused much discussion, both in the lay and scientific media, and prompted the development of position statements from international bone and mineral societies. A number of important issues have been raised (http://www.bmj.com/content/341/bmj.c3691/reply\#bmj el 240991), each of which will now be discussed.

\section{Cardiovascular events were not primary study endpoints}

A potential limitation of our meta-analysis is that cardiovascular outcomes were not the primary endpoints of any of the contributing studies (although they were prespecified secondary endpoints in two of the Auckland studies $[15,17])$, and consequently data on these outcomes were not gathered in a standardized manner. The events were obtained variously from self-reports, hospital admissions, death certificates, or adjudication of medical records. The RECORD study was the single largest contributor of participants, and diagnoses were based on self-reports of 
hospital admissions, and data from death certificates. The latter were the source for about $60 \%$ of MI, and $46 \%$ of strokes in that study. Two key facts address these concerns. First, the magnitude of the increased risk of MI with calcium was consistent across the trials irrespective of the method of event ascertainment. Secondly, the likelihood of differential misclassification or misreporting of cardiovascular events by treatment allocation is small, because the data came from blinded, placebo-controlled trials. The use of variable methods for collecting adverse event reports will introduce noise, not bias, and would tend to obscure a true effect rather than introduce a false one. This contention is supported by the results of the adjudication process of selfreported events from the Auckland Calcium Study, which changed the numbers of events in the two groups but not the asymmetry of their distribution [15]. It has also been suggested that failure of randomization may have produced an uneven distribution of risk factors for cardiovascular disease. This is unlikely across the number and size of studies included in the meta-analysis, and is not supported by examination of the baseline data from the calcium and placebo groups.

\section{Adverse effect restricted to subgroups}

Others have suggested that the effects might be related to trial- or participant-related characteristics, such as gender or type of calcium supplement used. However, there was no interaction between age, gender, baseline vitamin D status, or type of supplement used and risk of MI, stroke, or the composite endpoint with calcium (Figure 2). When the cohort was divided into 2 groups by baseline dietary calcium intake (above and below the median), there was an interaction between dietary calcium and the risk of MI (but not stroke or the composite endpoint) with calcium. But when the cohort was divided by quintile of baseline dietary calcium intake, there was no evidence of a dose-response relationship between baseline dietary calcium and the risk of $\mathrm{MI}$ with calcium, and in 4 of the 5 groups, the hazard ratios exceeded 1 and were similar to the hazard ratio for the entire cohort (Figure 2).

\section{No effect on mortality}

Some have questioned the internal consistency of the data since the increase in risk of $\mathrm{MI}$ is not accompanied by increased mortality. About $10-20 \%$ of individuals having an $\mathrm{MI}$ die as a result of that event, so the $30 \%$ increase in $\mathrm{Ml}$ found with calcium use should result in a 3-6\% increase in mortality. In fact, there is a $9 \%$ increase in mortality with calcium in the patient-level analysis and a $7 \%$ increase in the trial-level analysis, consistent with expectations. These results were not statistically significant, but the study did not have the power to detect differences of this magnitude. Hormone therapy and rosiglitazone are other examples of interventions that increase risk of $\mathrm{MI}$ to a similar degree as calcium supplements without evidence for a significant increase in mortality $[18,19]$

\section{Effect of calcium and vitamin D}

The exclusion of studies of co-administered calcium and vitamin D potentially limits the generalizability of the meta-analysis, since these agents are commonly taken together. However, the same could be said of the many other medications that are used in this age group and so will be co-administered with calcium, such as statins, aspirin and many more. This argument is only persuasive if a specific mechanism by which an agent might reverse the calcium effect can be identified, or if there are trial data suggesting an interaction. There is weak evidence that vitamin D might have an independent beneficial effect on mortality $[20,21]$ but unless this specifically prevents 
the adverse effect of calcium, patients taking both agents could still be worse off than those taking vitamin $\mathrm{D}$ alone. One of the studies included in the meta-analysis did compare calcium and vitamin D with vitamin D alone [22], and found risks of MI and stroke comparable to those obtained from analysis of studies of calcium monotherapy.

The Women's Health Initiative (WHI) analyzed the effects of calcium with vitamin D on vascular outcomes in 36,000 women followed for seven years [23]. The hazard ratio for $\mathrm{MI}$ or coronary heart disease death in the whole cohort was $1.04(95 \% \mathrm{Cl}$, 0.92-1.18), but for those without risk factors for coronary heart disease it was 1.19 $(95 \% \mathrm{Cl}, 0.97-1.59)$. For the composite endpoint of MI, coronary heart disease death, coronary artery bypass graft or percutaneous coronary intervention, the hazard ratio in the entire cohort suggested an adverse effect $(1.08,95 \% \mathrm{Cl}, 0.99-1.19)$. Vascular risk associated with randomization to calcium/vitamin $D$ was related to body mass index $(\mathrm{BMI})(\mathrm{P}=0.04)$ such that women with higher $\mathrm{BMI}$ were at lower risk from calcium/vitamin D supplementation [23]. For participants with BMI of 25-30, the hazard ratio for coronary heart disease was 1.18, and for BMI <25 it was 1.16. The overall conclusion of the WHI investigators was that intervention with calcium plus vitamin $\mathrm{D}$ did not change the risk of cardiovascular events.

The participants in the WHI differed in a number of respects from those in our recent meta-analysis. They were $~ 10$ years younger, heavier, $50 \%$ were taking hormone replacement therapy, and they had higher calcium intakes as a result of widespread use of non-trial calcium supplements. Over half of the WHI participants were taking calcium supplements at trial entry. We have now re-analyzed this study to determine whether non-protocol use of calcium supplements might have influenced the outcome. There are significant interactions between the effects of randomization to calcium-vitamin D on MI, stroke, and the combination of stroke or MI, and selfadministration of calcium $(0.006<P<0.045)$. In those not using non-protocol calcium supplements, there was a $\sim 20 \%$ increase in frequency of these events $(P=0.05)$ [24]. While this is not conclusive in its own right, it does suggest that the original analysis of cardiovascular events in WHI was misleading because it did not recognize the interaction with use of non-protocol calcium supplements. Once this is allowed for, the results appear to be comparable to those of the meta-analysis of trials of calcium monotherapy. Cardiovascular event data comparing subjects treated with calciumvitamin D and placebo are also available for the RECORD and Lappe [25] studies. When these are meta-analyzed with the WHI data for patients not self-medicating with calcium, essentially the same results as in the WHI reanalysis are found.

\section{Lower doses of calcium supplements might be adequate}

Some correspondents have pointed out that the mean total intake from dietary calcium and calcium supplements in the trials in our meta-analysis ( $1800 \mathrm{mg} / \mathrm{day})$ exceeds the recommended intake for postmenopausal women. It is important to note that clinical trials and observational data both suggest that the skeletal effects of calcium supplementation alone are small [26], and evidence of benefit has only been demonstrated in trials evaluating doses of calcium similar to those in the metaanalysis. In a recent meta-analysis of the effect of calcium supplements with or without vitamin D on fractures [27], 15 of the 16 studies with fracture as an endpoint administered at least $750 \mathrm{mg} /$ day of calcium supplement, and in 10 of the 13 studies that reported baseline dietary calcium intake, the total calcium intake (diet + 
supplements) was at least $1500 \mathrm{mg} / \mathrm{day}$ (range 1230-2300mg/day). There is no robust evidence that calcium supplements in doses $<1000 \mathrm{mg} /$ day or total calcium intake $<1500 \mathrm{mg} /$ day prevent fractures, and we have recently failed to detect any effect of a supplement of $600 \mathrm{mg} / \mathrm{day}$ on bone density in normal men [17]. Thus, the beneficial skeletal effects of calcium have only been demonstrated in trials evaluating the same doses of calcium that also increase risk of MI.

\section{Apparent conflict with other recent studies}

Wang et al recently published a meta-analysis of calcium effects on cardiovascular events, which appeared to be reassuring [28]. They reported a relative risk of 1.14 (95\% confidence interval 0.92-1.41) for cardiovascular events based on 3 randomized trials of calcium supplements. We found risks of similar magnitude for the composite of $\mathrm{MI}$, stroke, or sudden death in our patient-level analysis (hazard ratio 1.18) and trial-level analysis (relative risk 1.12). The consistency of results is not surprising because all of the trials included in the Wang meta-analysis were also included in our meta-analysis. However, our meta-analysis included more trials, captured more events, and therefore had greater statistical power than that of Wang. We also reported data for $\mathrm{Ml}$ and stroke separately.

Data for self-reported events provided by the investigators of a Perth study [29] were included in our meta-analysis, but these investigators have recently published analyses of hospital admission data from that study [30]. They do not provide data for $\mathrm{Ml}$ in this paper, but use a novel composite endpoint of atherosclerotic vascular mortality or first-hospitalisation, which includes diagnoses as diverse as arrhythmias and heart failure. The choice of such a diverse composite endpoint runs contrary to current recommendations [31]. The adjusted hazard ratio for this endpoint was 0.94 (95\% Cl 0.69-1.28). Despite the width of these confidence intervals and the low power of a single study of this size, they conclude that this analysis provides "compelling evidence" that calcium supplementation does not significantly increase the risk of vascular disease, whereas this confidence interval includes the hazard ratio found in the meta-analysis, albeit for a somewhat different endpoint. Further data presented in abstract form from this study indicate that calcium supplements may also increase the risk of admission to hospital with abdominal problems, which is a further cause for concern [32].

Osteoporosis treatments only work with calcium co-administration

It is frequently stated that approved therapies for osteoporosis are only effective when co-administered with calcium and vitamin D. This possibility has seldom been directly assessed, since this would require a randomized trial comparing the therapy plus calcium supplements, with the therapy administered alone. There is a randomized controlled trial of alendronate that directly addresses this question, and showed no difference in changes in bone density over two years from the addition of calcium supplementation to alendronate (Figure 3) [33]. It is also possible to compare changes in bone density between trials of zoledronate with or without calcium. The one-year change in spine bone density in a study not using calcium coadministration [34] was 4.8\%, in comparison with a change of $4.0 \%$ in the phase 3 trial which co-administered calcium and vitamin $D$ [35]. It is also sometimes stated that anti-fracture efficacy for osteoporosis treatments has not been demonstrated without co-administration of calcium and vitamin D. The McCloskey trial of clodronate [36] did not use either calcium or vitamin D and showed a comparable 
decrease in non-vertebral fracture to that found with much more potent antiresorptives, such as zoledronate [35] and denosumab [37], though hip fractures were not reduced in this unselected cohort. Also, anti-fracture efficacy of estrogen has been demonstrated without co-administration of calcium or vitamin $D$ [38]. While it would be desirable to have more extensive documentation of the effects of all of the therapeutic interventions used in the absence of calcium supplementation, the balance of current evidence suggests that calcium supplements do not substantially impact on the efficacy of these medications.

\section{Mechanisms of Calcium Supplement Effect on Vascular Disease}

The pathogenic mechanisms by which the use of calcium supplements increases the risk of cardiovascular events are open to speculation. It is noteworthy that there is no evidence of a positive relationship between dietary calcium intake and cardiovascular disease [39-41], though this is based on observational studies whose findings are often at odds with those from randomized trials, and few individuals in these studies had total calcium intakes $>1800 \mathrm{mg} /$ day, the median intake in the randomized trials in our meta-analysis. With these caveats, the absence of a signal from dietary calcium suggests that it is the use of a non-food supplement that increases cardiovascular risk, rather than simply the total calcium intake. Numerous studies have shown acute increases in serum calcium into the borderline hypercalcemic range, following ingestion of 500-1000 mg of calcium as a supplement [42-45] (Figure 4). This is in marked contrast to the ingestion of calcium-rich foods, which produce much smaller excursions in serum calcium [46], presumably because their transit through the upper gastrointestinal tract is much slower as a result of their significant contents of protein and fat. Thus, it is tempting to relate the cardiovascular effects of calcium supplements to the transient hypercalcemia that follows their administration.

There is an important related literature which considers the impact of high-normal levels of serum calcium on cardiovascular disease. Rubin [47] has shown that mean carotid artery plaque thickness is $50 \%$ greater in normal older Americans whose serum calcium is in the top quintile, when compared with those in the bottom quintile. Similarly, we have shown that for each $0.1 \mathrm{mmol} / \mathrm{L}$ increase in serum calcium, the likelihood of abdominal aortic calcification increases by $23 \%$, in a population of normal older women [48]. These changes in the arterial wall are paralleled by increases in cardiovascular events. Slinin [49] has shown that the combined incidence of stroke and coronary heart disease increases by $17 \%$ for each standard deviation increase in serum calcium in osteoporotic postmenopausal women. Lind demonstrated a 2.5 -fold increase in the risk of $\mathrm{Ml}$ across the measured range of calcium concentrations in 200050 -year old men followed for 18 years [50]. Foley has shown risk of stroke, but not coronary heart disease, to be related to serum calcium in a cohort of nearly 16,000 men and women, mean age 54 years, followed for 13 years [51]. In a retrospective study of 13,000 men, serum calcium was a predictor of myocardial infarction after adjustment for other cardiovascular risk factors, with an odds ratio of 1.2 per $0.1 \mathrm{mmol} / \mathrm{L}$ increase in serum calcium $(P<0.0001)$ [52]. In normal Swedish men aged under 50, Leifsson has shown a steep relationship between 10-year mortality (predominantly cardiovascular) and baseline serum calcium [53]. In this study, a serum calcium between 2.50 and $2.55 \mathrm{mmol} / \mathrm{L}$ conferred a 50\% increase in mortality compared with a calcium between 2.30 and $2.45 \mathrm{mmol} / \mathrm{L}$. Similarly, primary hyperparathyroidism has been associated with increased risk of vascular events [54]. 
Thus, a simple explanation of the calcium supplement effect would be that a supplement moves an individual into the upper range of serum calcium for up to six hours following each dosing, and thus increases their cardiovascular risk. Indeed, the increase in ionized calcium of $0.08 \mathrm{mmol} / \mathrm{L}$ that has been observed after supplement ingestion $[42,43]$ would equate to an increase in cardiovascular event rates of $27 \%$, based on the data in the Slinin study. This is very similar to what was observed in the meta-analysis, which is surprising since the increase in serum calcium following calcium supplements lasts only about 6 hours, though many individuals take more than one supplement daily. Possibly it is peak rather than mean levels of calcium which drive the adverse cardiovascular effects. If the acute changes in serum calcium following supplement ingestion are critically important, then it would also be important to determine the effect of taking a calcium supplement with or immediately after a meal. This would be expected to blunt the excursion in serum calcium, so it could be hypothesized that this might also reduce the adverse cardiovascular effects of calcium supplements.

If higher levels of serum calcium increase vascular risk, then the mechanisms of this still remain to be determined. The documentation of increased carotid artery plaque thickness and abdominal aortic calcification suggest a vessel wall effect. Similarly, in patients with renal impairment, acceleration of coronary artery calcification associated with calcium supplement use has been clearly documented [13]. If this is what indeed occurs, then it is unlikely to be a simple physicochemical process, since vascular calcification is a regulated, cell-mediated phenomenon [55]. Pyrophosphate is an important inhibitor of tissue calcification which also complexes with calcium, so its levels would be expected to be reduced when extracellular fluid calcium concentrations rise. We have measured two other regulators of vascular calcification, osteoprotegerin and matrix gla protein, in subjects taking calcium supplements, but failed to show any difference from controls [56]. The effect of increased calcium concentrations could be directly on the cells of the vessel wall, mediated through the calcium sensing receptor. This possibility is supported by the demonstration in vitro that matrix vesicle formation and calcium incorporation into cultures of vascular smooth muscle cells are both increased at higher media calcium concentrations [57] and the fact that polymorphisms of the calcium sensing receptor are associated with cardiovascular mortality [58]. Increased circulating calcium concentrations will also reduce parathyroid hormone (PTH) levels, and PTH has been shown to inhibit arteriosclerotic Wnt/ $\beta$-catenin signaling in vascular smooth muscle cells [59].

The time-course of the cardiovascular events in the meta-analysis may provide some insights into pathogenesis. Much of the increase in risk of myocardial infarction occurs within the first year, though there is a continued divergence of the two groups on the Kaplan-Meier plot subsequently. In contrast, the divergence of stroke incidence between the two groups only begins at about one year and progressively increases subsequently. This difference in time-course could reflect the different size of arteries involved in myocardial infarction and stroke, or it could indicate that a mechanism other than progressive vascular calcification is contributing to the early increase in myocardial infarction. One such possibility would be the induction of a hypercoagulable state associated with increased serum calcium. Since calcium is integral to many stages of the coagulation process and to platelet function, such a possibility is plausible. Indeed, it has been demonstrated that the acute induction of 
severe hypercalcemia in rats is associated with a 50\% reduction in clotting time [60]. That this might also occur with more physiological perturbations of circulating calcium is suggested by an in vitro study of human blood in which the time to the initiation of coagulation is found to be inversely related to ionized calcium concentration, over a range from 0.6 to $1.7 \mathrm{mmol} / \mathrm{L}$ (Figure 5) [61].

A third mechanism by which increases in serum calcium could have an early effect on cardiovascular morbidity is by way of its effects on arterial stiffness. This has been assessed in several contexts. Studies in patients with mild primary

hyperparathyroidism have shown impaired indices of vasodilatation [62, 63]. In patients undergoing hemodialysis, the induction of mild hypercalcemia, through use of a high-calcium dialysate, results in acute increases in arterial stiffness assessed by digital photoplethysmography [64]. Supportive data comes from studies of normal volunteers in whom increases in serum ionized calcium of $0.3 \mathrm{mmol} / \mathrm{L}$ increase systolic blood pressure (mean $\pm S D, 114 \pm 13$ to $121 \pm 10 \mathrm{mmHg} ; \mathrm{P}<0.05$ ) and result in dose-related impairment in endothelial vasodilator function [65].

\section{Conclusions}

Our recent meta-analysis raises the possibility that calcium supplements increase cardiovascular risk. This finding is consistent with clinical trials of calcium supplements in patients with renal impairment, and with epidemiological data relating circulating calcium concentrations to cardiovascular disease in normal populations. There are a number of plausible pathophysiological mechanisms for these effects.

A key question that then arises is whether the size of this adverse effect is large enough to negate the benefit of calcium use for skeletal health - the likelihood that calcium increases vascular events needs to be balanced against its only modest effects on fracture rates. When the change in number of events is calculated from the meta-analysis data, we find that treatment of 1000 women with calcium for 5 years results in 14 more Mls, 10 more strokes and 13 more deaths, while reducing the number of fractures by 26 [16]. These analyses need to be repeated using populations of different ages with different risk profiles, to determine how generalizable these findings are. However, we have carried out a similar analysis in the low-risk population of the WHI, and have also found that the increased cardiovascular risk negates the small benefit from fracture prevention. How the riskbenefit is influenced by differences in the dose of calcium and vitamin $D$ also needs further study. Based on the best evidence available, it seems likely that there is no net benefit from use of calcium supplements, and there may well be net detriment. Since food sources of calcium produce similar benefits on bone density as supplements $[66,67]$ (although effects on fracture are unclear), and have not been associated with adverse cardiovascular effects, these may be a better option as the principal calcium source. This suggestion is supported by the fact that calcium-rich meals have little effect on serum calcium concentrations. A move away from supplements may make lower target calcium intakes necessary, since $>1000 \mathrm{mg} /$ day may be difficult for many patients to achieve. Optimization of vitamin D status [68] to facilitate absorption of dietary calcium remains a sensible measure for the maintenance of bone health.

The borderline anti-fracture efficacy of calcium means that it should not be relied on as the primary therapy in anyone with a substantially increased fracture risk - such 
patients require therapies proven to prevent fractures. Available evidence suggests that these therapies will be effective without co-administration of calcium supplements. 


\section{Legends to Figures}

\section{Figure 1}

Effect of calcium supplements on risk of myocardial infarction from an individual patient meta-analysis of 5 trials (8151 participants), and a trial level analysis of 11 studies (11921 subjects). Significant increases in risk were shown for both analyses. Figure from Bolland et al [16], used with permission.

\section{Figure 2}

Effect of calcium supplementation on myocardial infarction (MI) in participant subgroups. Data are from the meta-analysis of Bolland et al [16], and are drawn from the 5 studies that contributed patient-level data.

\section{Figure 3}

Changes in bone mineral density in the lumbar spine $(A)$ and femoral neck $(B)$ in postmenopausal women with low bone density randomized to treatment with alendronate, alendronate plus calcium, or calcium alone, over 2 years. Asterisks denote differences between the alendronate and calcium groups, and daggers differences between the alendronate+calcium and calcium groups, respectively. There were $\mathrm{n}$ differences between the alendronate alone and alendronate+calcium groups. From Bonnick et al ) [33], used with permission.

\section{Figure 4}

Changes in serum ionized calcium concentration following administration of control (squares), calcium citrate (triangles), calcium carbonate (asterisks), or potassium citrate (circles). The calcium doses were both $1 \mathrm{~g}$. $\dagger$ denotes a significant difference from control, and $\ddagger$ denotes difference from potassium citrate. From Karp et al [43], used with permission.

\section{Figure 5}

Relationship between the time to the initiation of coagulation (r-time) and ionized calcium concentrations in in vitro studies of human blood samples. For ionized calcium values above $0.56 \mathrm{mmol} / \mathrm{L}$, the correlation with r-time was $-0.48(\mathrm{P}<0.001)$. From James et al [61], used with permission.

\section{Figure 6}

Possible sequence of events that might contribute to the acceleration of vascular disease by calcium supplementation. As discussed in the text, there is evidence for each component shown in this pathway, mostly from observational studies in normal cohorts stratified by baseline serum calcium level, but there may be other pathways and other intermediate steps that are not yet known. Meta-analysis of trial data show an increase in cardiovascular events, but not in mortality, though increased mortality has been shown to be associated with high-normal serum calcium concentrations in a prospective observational study. Copyright IR Reid, used with permission.

\section{Acknowledgements}

The authors are grateful to Dwight A. Towler for valuable discussions of the pathogenesis of vascular disease, and to Sarah Bristow for help in writing this manuscript. 


\section{References}

1. Yacowitz H, Fleischman Al, Bierenbaum ML (1965) Effects of oral calcium on serum lipids in man. BMJ 1:1352-1354

2. Govers MJAP, Vandermeer R (1993) Effects of dietary calcium and phosphate on the intestinal interactions between calcium, phosphate, fatty acids, and bile acids. Gut 34:365-370

3. Denke MA, Fox MM, Schulte MC (1993) Short-term dietary calcium fortification increases fecal saturated fat content and reduces serum lipids in men. $J$ Nutr 123:1047-1053

4. Christensen R, Lorenzen JK, Svith CR, Bartels EM, Melanson EL, Saris WH, Tremblay A, Astrup A (2009) Effect of calcium from dairy and dietary supplements on faecal fat excretion: a meta-analysis of randomized controlled trials. Obesity Reviews 5. Zemel MB, Sun X (2008) Calcitriol and energy metabolism. Nutrition Reviews 66:S139-S146

6. Reid IR, Mason B, Horne A, Ames R, Clearwater J, Bava U, Orr-Walker B, Wu F, Evans MC, Gamble GD (2002) Effects of calcium supplementation on serum lipid concentrations in normal older women: A randomized controlled trial. Am J Med 112:343-347

7. Bostick RM, Fosdick L, Grandits GA, Grambsch P, Gross M, Louis TA (2000) Effect of calcium supplementation on serum cholesterol and blood pressure - A randomized, double-blind, placebo-controlled, clinical trial. Arch Family Med 9:31-38 8. Reid IR, Ames R, Mason B, Bolland MJ, Bacon CJ, Reid HE, Kyle C, Gamble GD, Grey A, Horne A (2010) Effects of calcium supplementation on lipids, blood pressure, and body composition in healthy older men: a randomized controlled trial. Am J Clin Nutr 91:131-139

9. Griffith LE, Guyatt GH, Cook RJ, Bucher HC, Cook DJ (1999) The influence of dietary and nondietary calcium supplementation on blood pressure -An updated metaanalysis of randomized controlled trials. Am J Hypertens 12:84-92

10. Reid IR, Horne A, Mason B, Ames R, Bava U, Gamble GD (2005) Effects of calcium supplementation on body weight and blood pressure in normal older women: A randomized controlled trial. J Clin Endocrinol Metab 90:3824-3829

11. van Mierlo LAJ, Arends LR, T Streppel M, Zeegers MPA, Kok FJ, Grobbee DE, Geleijnse JM (2006) Blood pressure response to calcium supplementation: a meta-analysis of randomized controlled trials. J Human Hypertens 20:571-580

12. Lanou AJ, Barnard ND (2008) Dairy and weight loss hypothesis: an evaluation of the clinical trials. Nutrition Reviews 66:272-279

13. Russo D, Miranda I, Ruocco C, Battaglia Y, Buonanno E, Manzi S, Russo L, Scafarto A, Andreucci VE (2007) The progression of coronary artery calcification in predialysis patients on calcium carbonate or sevelamer. Kidney Int 72:1255-1261 14. Block GA, Raggi P, Bellasi A, Kooienga L, Spiegel DM (2007) Mortality effect of coronary calcification and phosphate binder choice in incident hemodialysis patients. Kidney Int 71:438-441

15. Bolland MJ, Barber PA, Doughty RN, Mason B, Horne A, Ames R, Gamble GD, Grey A, Reid IR (2008) Vascular events in healthy older women receiving calcium supplementation: randomised controlled trial. BMJ 10.1136/bmj.39440.525752.BE:

16. Bolland MJ, Avenell A, Baron JA, Grey A, MacLennan GS, Gamble GD, Reid IR (2010) Effect of calcium supplements on risk of myocardial infarction and cardiovascular events: meta-analysis - art. no. c3691. British Medical Journal, 341: C3691-C3691 Jul 292010 
17. Reid IR, Ames R, Mason B, Reid HE, Bacon CJ, Bolland MJ, Gamble GD, Grey A, Horne A (2008) Randomized controlled trial of calcium supplementation in healthy, nonosteoporotic, older men. Arch Int Med 168:2276-2282

18. Writing Group for the Women's Health Initiative Investigators (2002) Risks and benefits of estrogen plus progestin in healthy postmenopausal women. JAMA 288:321-333

19. Nissen SE, Wolski K (2010) Rosiglitazone Revisited. Arch Int Med 170:11911201

20. Autier P, Gandini S (2007) Vitamin D supplementation and total mortality: a meta-analysis of randomized controlled trials. Arch Int Med 167:1730-1737

21. Melamed ML, Michos ED, Post W, Astor B (2008) 25-hydroxyvitamin D levels and the risk of mortality in the general population. Arch Int Med 168:1629-1637

22. Grant AM, Avenell A, Campbell MK, et al. (2005) Oral vitamin D3 and calcium for secondary prevention of low-trauma fractures in elderly people (Randomised Evaluation of Calcium Or vitamin D, RECORD): a randomised placebo-controlled trial. Lancet 365:1621-1628

23. Hsia J, Heiss G, Ren H, et al. (2007) Calcium/vitamin D supplementation and cardiovascular events. Circulation 115:846-854

24. Bolland MJ, Grey A, Gamble GD, Reid IR (2010) Risk of cardiovascular events with calcium/vitamin D - a re-analysis of the Women's Health Initiative. J Bone Miner Res 25 (suppl 1):S50

25. Lappe JM, Travers-Gustafson D, Davies KM, Recker RR, Heaney RP (2007) Vitamin $\mathrm{D}$ and calcium supplementation reduces cancer risk: results of a randomized trial. Am J Clin Nutr 85:1586-1591

26. Bischoff-Ferrari HA, Dawson-Hughes B, Baron JA, et al. (2007) Calcium intake and hip fracture risk in men and women: a meta-analysis of prospective cohort studies and randomized controlled trials. Am J Clin Nutr 86:1780-1790

27. Tang BM, Eslick GD, Nowson C, Smith C, Bensoussan A (2007) Use of calcium or calcium in combination with vitamin $D$ supplementation to prevent fractures and bone loss in people aged 50 years and older: a meta-analysis. Lancet 370:657-666

28. Wang L, Manson JE, Song Y, Sesso HD (2010) Systematic review: Vitamin D and calcium supplementation in prevention of cardiovascular events. Annals of internal medicine 152:315-323

29. Prince RL, Devine A, Dhaliwal SS, Dick IM (2006) Effects of calcium supplementation on clinical fracture and bone structure - Results of a 5-year, doubleblind, placebo-controlled trial in elderly women. Arch Int Med 166:869-875

30. Lewis JR, Calver J, Zhu K, Flicker L, Prince RL (2010) Calcium supplementation and the risks of atherosclerotic vascular disease in older women: results of a 5-year RCT and a 4.5-year follow-up. J Bone Miner Res published online: 31. Montori VM, Permanyer-Miralda G, Ferreira-Gonzalez I, et al. (2005) Validity of composite end points in clinical trials. BMJ 330:594-596

32. Lewis JR, Zhu K, Prince RL (2010) Errors in self-reported myocardial infarction in calcium intervention studies. Proceedings of the Australian \& New Zealand Bone \& Mineral Society Annual Scientific Meeting P17

33. Bonnick S, Broy S, Kaiser F, Teutsch C, Rosenberg E, DeLucca P, Melton M (2007) Treatment with alendronate plus calcium, alendronate alone, or calcium alone for postmenopausal low bone mineral density. Curr Med Res Opin 23:1341-1349

34. Grey A, Bolland MJ, Wattie D, Horne A, Gamble G, Reid IR (2009) The antiresorptive effects of a single dose of zoledronate persist for two years: a 
randomized, placebo-controlled trial in osteopenic postmenopausal women. J Clin Endocrinol Metab 94:538-544

35. Black DM, Delmas PD, Eastell R, et al. (2007) Once-yearly zoledronic acid for treatment of postmenopausal osteoporosis. N Engl J Med 356:1809-1822

36. McCloskey EV, Beneton M, Charlesworth D, et al. (2007) Clodronate reduces the incidence of fractures in community-dwelling elderly women unselected for osteoporosis: results of a double-blind, placebo-controlled randomized study. J Bone Miner Res 22:135-141

37. Cummings SR, San Martin J, McClung MR, et al. (2009) Denosumab for Prevention of Fractures in Postmenopausal Women with Osteoporosis. N Engl J Med 361:756-765

38. Lindsay R, Hart DM, Forrest C, Baird C (1980) Prevention of spinal osteoporosis in oophorectomised women. Lancet ii:1151-1153

39. Bostick RM, Kushi LH, Wu Y, Meyer KA, Sellers TA, Folsom AR (1999)

Relation of calcium, vitamin $\mathrm{D}$, and dairy food intake to ischemic heart disease mortality among postmenopausal women. Am J Epidemiol 149:151-161

40. Knox EG (1973) Ischaemic-heart-disease mortality and dietary intake of calcium. Lancet ii:1465-1467

41. Gibson RA, Makrides M, Smithers LG, Voevodin M, Sinclair AJ (2009) The effect of dairy foods on CHD: a systematic review of prospective cohort studies. British Journal of Nutrition 102:1267-1275

42. Reid IR, Schooler BA, Hannon S, Ibbertson HK (1986) The acute biochemical effects of four proprietary calcium supplements. Aust N Z J Med 16:193-197

43. Karp HJ, Ketola ME, Lamberg-Allardt CJE (2009) Acute effects of calcium carbonate, calcium citrate and potassium citrate on markers of calcium and bone metabolism in young women. British Journal of Nutrition 102:1341-1347

44. Heller HJ, Stewart A, Haynes S, Pak CYC (1999) Pharmacokinetics of calcium absorption from two commercial calcium supplements. J Clin Pharmacol 39:11511154

45. Heller HJ, Greer LG, Haynes SD, Poindexter JR, Pak CYC (2000) Pharmacokinetic and pharmacodynamic comparison of two calcium supplements in postmenopausal women. J Clin Pharmacol 40:1237-1244

46. Green JH, Booth C, Bunning R (2003) Postprandial metabolic responses to milk enriched with milk calcium are different from responses to milk enriched with calcium carbonate. Asia Pacific Journal of Clinical Nutrition 12:109-119

47. Rubin MR, Rundek T, McMahon DJ, Lee H-S, Sacco RL, Silverberg SJ (2007) Carotid artery plaque thickness is associated with increased serum calcium levels: the Northern Manhattan study. Atherosclerosis 194:426-432

48. Bolland MJ, Wang TKM, van Pelt NC, Horne AM, Mason BH, Ames RW, Grey AB, Ruygrok PN, Gamble GD, Reid IR (2010) Abdominal Aortic Calcification on Vertebral Morphometry Images Predicts Incident Myocardial Infarction. J Bone Miner Res 25:505-512

49. Slinin Y, Blackwell T, Ishani A, Cummings SR, Ensrud KE (2010) Serum calcium, phosphorus and cardiovascular events in post-menopausal women. Int J Cardiol (in press):

50. Lind L, Skarfors E, Berglund L, Lithell H, Ljunghall S (1997) Serum calcium: a new, independent, prospective risk factor for myocardial infarction in middle-aged men followed for 18 years. J Clin Epidemiol 50:967-973 
51. Foley RN, Collins AJ, Ishani A, Kalra PA (2008) Calcium-phosphate levels and cardiovascular disease in community-dwelling adults: the Atherosclerosis Risk in Communities (ARIC) Study. Am Heart J 156:556-563

52. Jorde R, Sundsfjord J, Fitzgerald P, Bonaa KH (1999) Serum calcium and cardiovascular risk factors and diseases: the Tromso study. Hypertension 34:484490

53. Leifsson BG, Ahren B (1996) Serum calcium and survival in a large health screening program. J Clin Endocrinol Metab 81:2149-2153

54. Nilsson IL, Yin L, Lundgren E, Rastad J, Ekbom A (2002) Clinical presentation of primary hyperparathyroidism in Europe -Nationwide cohort analysis on mortality from nonmalignant causes. J Bone Miner Res 17:N68-N74

55. Persy V, D'Haese P (2009) Vascular calcification and bone disease: the calcification paradox. Trends in Molecular Medicine 15:405-416

56. Reid IR, Bolland MJ, Grey A (2010) Does Calcium Supplementation Increase Cardiovascular Risk? Clin Endocrinol

57. Reynolds JL, Joannides AJ, Skepper JN, Mcnair R, Schurgers LJ, Proudfoot D, Jahnen-Dechent W, Weissberg PL, Shanahan CM (2004) Human Vascular Smooth Muscle Cells Undergo Vesicle-Mediated Calcification in Response to Changes in Extracellular Calcium and Phosphate Concentrations: A Potential Mechanism for Accelerated Vascular Calcification in ESRD. J Am Soc Nephrol 15:2857-2867

58. Marz W, Seelhorst U, Wellnitz B, Tiran B, Obermayer-Pietsch B, Renner W, Boehm BO, Ritz E, Hoffmann MM (2007) Alanine to serine polymorphism at position 986 of the calcium-sensing receptor associated with coronary heart disease, myocardial infarction, all-cause, and cardiovascular mortality. J Clin Endocrinol Metab 92:2363-2369

59. Cheng SL, Shao JS, Halstead LR, Distelhorst K, Sierra O, Towler DA (2010) Activation of Vascular Smooth Muscle Parathyroid Hormone Receptor Inhibits Wnt/beta-Catenin Signaling and Aortic Fibrosis in Diabetic Arteriosclerosis. Circulation Research 107:271-U222

60. Hilgard P (1973) Experimental hypercalcaemia and whole blood clotting. J Clin Path 28:616-619

61. James MFM, Roche AM (2004) Dose-response relationship between plasma ionized calcium concentration and thrombelastography. Journal of Cardiothoracic and Vascular Anesthesia 18:581-586

62. Neunteufl T, Katzenschlager R, Abela C, Kostner K, Niederle B, Weidinger F, Stefenelli T (1998) Impairment of endothelium-independent vasodilation in patients with hypercalcemia. Cardiovascular Research 40:396-401

63. Baykan M, Erem C, Erdogan T, Hacihasanoglu A, Gedikli O, Kiris A, Kucukosmanoglu M, Ersoz HO, Celik S (2007) Impairment of flow mediated vasodilatation of brachial artery in patients with primary hyperparathyroidism. International Journal of Cardiovascular Imaging 23:323-328

64. Kyriazis J, Katsipi I, Stylianou K, Jenakis N, Karida A, Daphnis E (2007) Arterial stiffness alterations during hemodialysis: The role of dialysate calcium. Nephron Clinical Practice 106:C34-C42

65. Nilsson IL, Rastad J, Johansson K, Lind L (2001) Endothelial vasodilatory function and blood pressure response to local and systemic hypercalcemia. Surgery 130:986-990 
66. Lau EMC, Woo J, Lam V, Hong A (2001) Milk supplementation of the diet of postmenopausal Chinese women on a low calcium intake retards bone loss. J Bone Miner Res 16:1704-1709

67. Cadogan J, Eastell R, Jones N, Barker ME (1997) Milk intake and bone mineral acquisition in adolescent girls - randomised, controlled intervention trial. BMJ 315:1255-1260

68. Committee to Review Dietary Reference Intakes for Vitamin $D$ and Calcium (2010) Dietary Reference Intakes for Calcium and Vitamin D. Institue of Medicine, Washington DC 